\title{
Segmentation-based Image Analysis Tool for Surveying and Statistical Applications
}

\author{
Tanvir Ahmed ${ }^{1}$, Muhammad Shafiul Munir ${ }^{2}$ and Shoeb Ahmed ${ }^{3 *}$ \\ Department of Chemical Engineering, \\ Bangladesh University of Engineering and Technology, \\ Dhaka-1000, Bangladesh \\ 1'tanvirche32@gmail.com, ${ }^{2}$ shafi.munir@gmail.com, \\ ${ }^{3}$ shoebahmed@che.buet.ac.bd
}

\begin{abstract}
With the advancement of modern technology, imaging has become a significant inventory with a great range of applications throughout medical science, biological research and industrial safety; if analyzed properly. However, considering the flexibility and appropriateness for varying applications most of the image processing methods do not fit in as suitable. Considering simplicity, widespread applicability and customization options image analysis using MATLAB-based custom codes has already gained popularity in many perspectives. One of the most promising applications where image analysis can cater the requirements of the user faster and with a lesser investment would be Geographical surveying. This work is based on statistical analysis of satellite map using MATLAB to exhibit how a customized application can perform separate clear mapping of various objects and areas, specifications of different terrain properties and statistical surveying of the region of interest. This program takes image from satellite map as input, segments the image in various maps like road map, field map etc. and provides necessary statistical data for each segments. It can be developed to address any further need as well as can be applied in different color-based image analysis applications if required. However, coding knowledge and techniques being unfamiliar to many people, might lessen its user-friendliness too some extent. To overcome this limitation a graphical interface of the program has been developed for the users possessing no MATLAB or computational language experience. The program is also customized to work on satellite map snapshots in different zooms. Wide range of applications of the program include geographical survey, surveillance and tracking, urban planning and design etc.
\end{abstract}

Keywords: Geographical survey, GUI, Image processing, Image segmentation

\section{Introduction}

Analysis of imagery of various format and form has been an essential tool in analyzing and evaluating various geographical and biological images to extract information from them in order to utilize in further studies [1-4]. In medical and biological sciences, image analysis has been proven extremely significant to elaborate the diverse images taken from laboratory experiments, internal and external body parts in diagnosis and treatment of diseases [1, 5-8]. It is also important in geological survey and research, considering the requirement of analysis of geographical maps and images repeatedly [9-13]. Systematic image analysis also provides valuable data extracted from images to be utilized in statistical analysis $[7,14,15]$. There being many available tools for analyzing images;

Received (April 24, 2017), Review Result (November 24, 2017), Accepted (March 6, 2018)

${ }^{*}$ Corresponding Author 
most of them lacks universal suitability, appropriateness and flexibility for varying applications. Computational language-based image analysis approach offers the flexibilities without the limitations of the commercial software. Because of the simplicity, widespread applicability and customization options, image analysis using MATLABbased custom codes has already gained popularity in many perspectives [7, 16-18]. Wide range of images can be analyzed using algebraic theories and functions building up particular unique algorithm for each task. The main feature that makes MATLAB the most ideal tool is its capability to address any sorts of needs due to its unparalleled flexibility and its functional inventory, which is able to provide diversified methods to deal with problems within a great range.

Nowadays, satellite and mapping systems can provide large volumes of data that are invaluable in monitoring earth resources and the impacts of human activities. Images obtained by satellites are useful in many environmental applications such as geographical mapping, prediction of agricultural crops, urban growth, weather, flood and fire control etc., $[9,13]$. Analysis of high resolution satellite images has been an important tool to acquire accurate and up-to-date road network information essential for urban planning. One of the major information that can be extracted from the images obtained from satellite map is the roads that constitute an important layer of vector maps. However, along with roads, field, water and residential areas are also the points of interest to different users. Road network information is required for a variety of applications. Manual extraction of roads and other zones directly from satellite map is very timeconsuming and not straight forwards. It becomes even more complicated for road network in regions that consists of larger parts of desert or dry mountainous areas, e.g., northern Africa. Because of the complexity and considering the volume of work, it is convenient to use automated image analysis approaches [10]. These extraction methods can be of many different forms according to purposes, sources and applications of the images. Considering simplicity, widespread applicability and customization options image analysis using MATLAB-based custom codes has already gained popularity in many perspectives. Here, a MATLAB-based approach has been proposed and implemented for detailed statistical analysis of satellite maps. A typical satellite map was analyzed using MATLAB to exhibit how a customized application can perform individual mapping of various objects and areas, measurement of different terrain properties and statistical surveying of the region of interest.

\section{Image Analysis with Matlab}

There are quite a few of studies reported regarding image analysis techniques. Some of them explained the basic techniques behind the image analysis methods while others showed various functions and tools of analysis. Mangala et al., proposed a new automatic road extraction technique using gradient operation and skeletal ray formation [12]. Considering the usage of satellite images and the significance of road extraction from imagery the authors found a more suitable and effective way to isolate road from satellite images. Jens Rittscher reviewed necessary image analysis techniques as well as applied these techniques to deliver the data that will enable systems-level biology [6]. Some recent studies demonstrated another significant facet of image analysis to parse intracellular signaling during cell migration, a hallmark of cancer cell [7, 16, 18]. Application of MATLAB in image analysis makes it more convenient, customized and affordable, thus making it one of the most popular approaches for analyzing industrial, medical and biological images.

Image segmentation can be classified in to three categories such as Threshold technique, Region-based image segmentation, and Edge-based image segmentation [19, 20]. The threshold technique is the simplest of segmenting methods. If an image $f(x, y)$ is composed of light objects on a dark background, in such a way that object and 
background pixels have intensity levels grouped into two dominant modes, one obvious way to extract the objects from the background is to select a threshold $\mathrm{T}$ that separates these modes. Then any point $(x, y)$ for which $f(x, y) \geq T$ is called an object point; otherwise the point is called a background point. In other words, the thresholded image $g(x, y)$ is defined as

$$
g(x, y)=\left\{\begin{array}{l}
1 ; f(x, y) \geq T \\
0 ; f(x, y)<T
\end{array}\right.
$$

Data clustering is one method of Region-based image segmentation, and it is popularly used in mathematics and statistics. Centroids or prototypes are used to present the great numbers of cluster to achieve the two goals of reducing the computational time and providing a better condition to compress it [19]. Edge detection and corner detection are getting utilized recently in digital image processing. The watershed image segmentation is an example of edge-based image segmentation [19].

Justyna Inglot discussed the basic uses and applications of MATLAB in image processing and segmentation [17]. Graphical User Interface mostly known as GUI is important to present the complicated codes in an easily understandable, executable format which is essential for the non-technical people to be able to use it. Sharma et al. summarize the automated segmentation methods, specifically discussed in the context of Computed topography (CT) and Magnetic resonance (MR) images (8). Accurate segmentation of medical images is a key step in contouring during radiotherapy planning. $\mathrm{CT}$ and MR imaging are the most widely used radiographic techniques in diagnosis, clinical studies and treatment planning. Cloppet et al., proposed a new segmentation method of complex nucleus configurations [1]. The developed method is based on the marker based watershed algorithm which is one of the most widely accepted solutions in biomedical imaging. Gonzalez et al., summarizes substantial amount of works and techniques for image segmentation [21].

\section{Methodology}

The most prominent aspect of the approach is that it is relatively flexible and open to changes according to the needs and is quite user friendly despite being totally dependent on MATLAB programming. Basic steps are shown in Figure 1. This program takes satellite map as an input and segregate the regions to shows segmented road, field and water area maps as necessary. It also provides quantitative information of the region as bar and pie charts. Raw values are stored in excel files by default. Because of the flexible nature of the code it is able to provide more information extracted from the original map if designed by the user based on requirements.

The program has two sections designed for two different situations; one for zoomed out maps and another for zoomed in maps. Two separate parts are displayed in simple graphical user interfaces $[22,23]$ which indicates that in order to operate the program the user does not need to have MATLAB basics. The main algorithm is also different for two types of maps. For the zoomed in ones the input image is processed through intensity based thresholding followed by various spatial filters using specificity based on particular property geometry. The processed image is then further undergone through some morphological operations to obtain the desired output map. In case of the zoomed out ones after extracting the individual maps of roads, fields, water ways and residential areas through intensity based segmentation utilizing the scaling factor in the map the area of individual areas are found out through algebraic equations. 


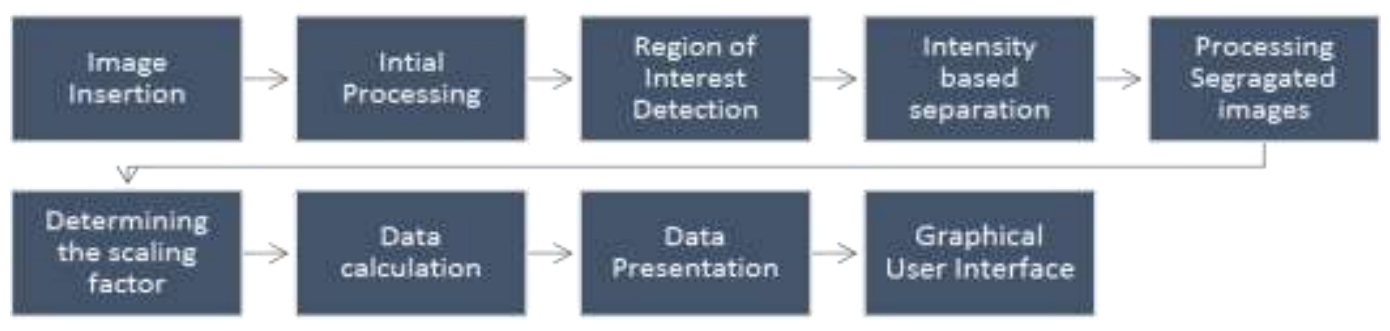

Figure 1. Flowchart of Satellite Map Segmentation and Statistical Analysis

Finally, the segmented images and extracted data are presented in pie chart, bar chart and excel sheet for further calculation and analysis. The calculation and data extraction has been done only for the second part but it can be performed for the first part as well by modifying the code.

The simple steps to run the program are as follows:

1. As the user starts the program a graphical user interface will appear exactly like Figure 2(a).

2. User needs to insert two pictures

$i$. As 'input image'- the satellite map on which the program would operate

ii. As 'scale image'- image containing the scale bar of the bottom left corner of satellite map

3. User needs to specify the requirements entering 'code number' indicating what kind of output is desired

4. The value that the scale bar represents needs to be inserted as scaling factor.

5. Once all the required information are provided the 'OK' button needs to be pressed to initiate the processing. This will complete the image analysis and show the desired outputs.

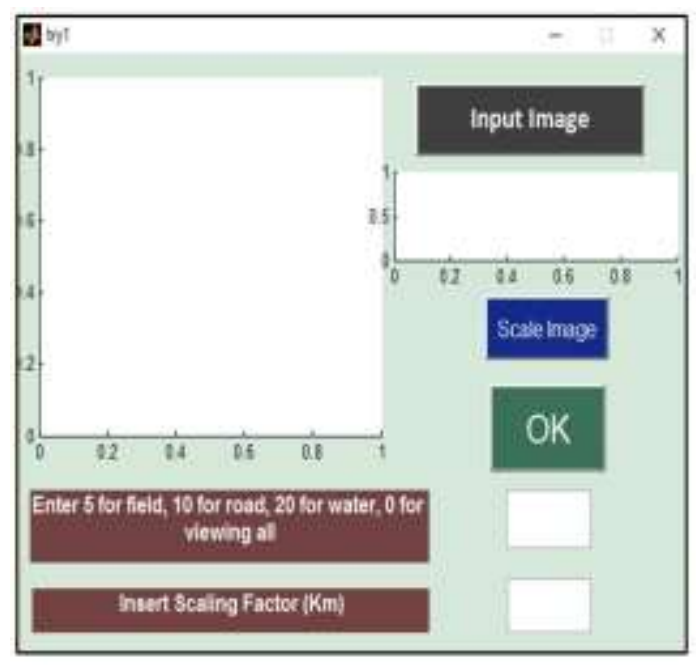

(a)

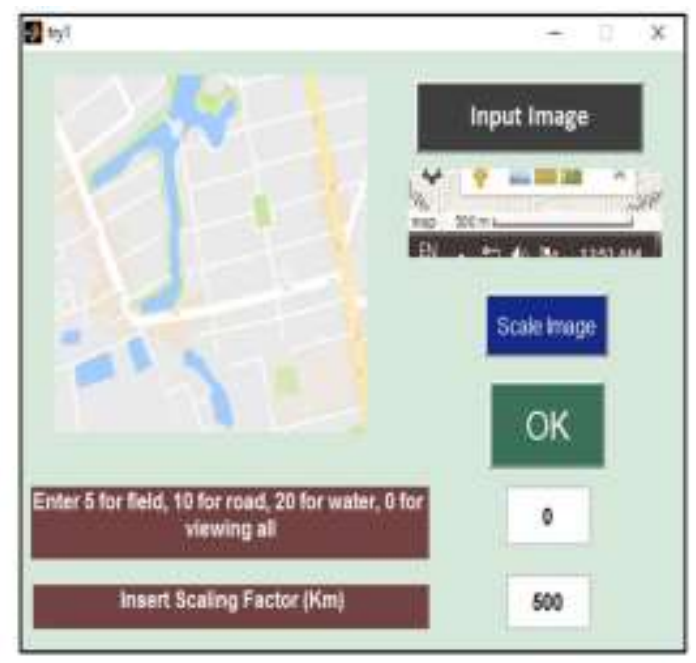

(b)

Figure 2. (a) Graphical User Interface for Satellite Map Segmentation; (b) Graphical User Interface after Giving Input 


\section{Results and Discussion}

The program is designed to generate various maps from the input images. The currently designed code can generate four types of segmented maps from satellite map containing only roads, fields, water ways and residential areas. Graphical interface, as shown in Figure 2 dictates the segmentation options of the figure as well.

a)

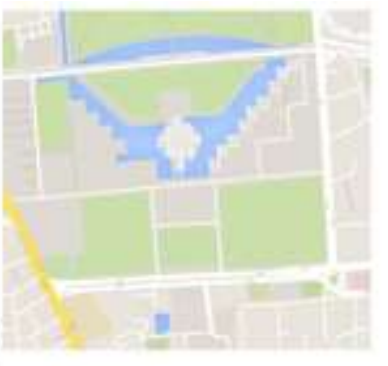

b)

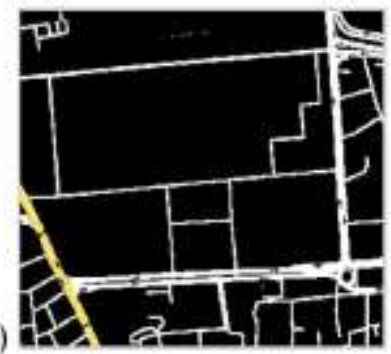

c)

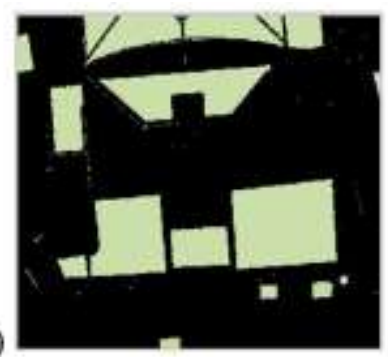

d)

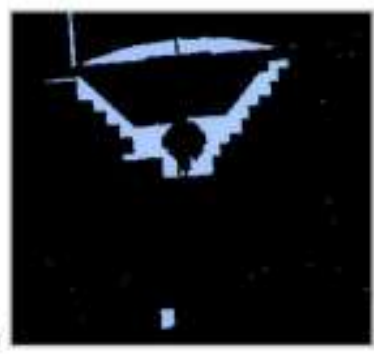

e)

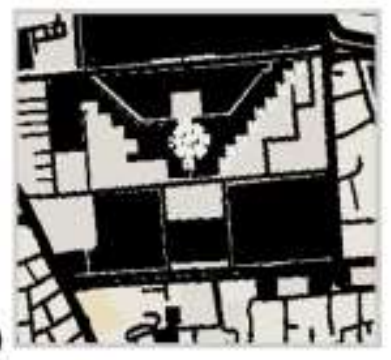

f)

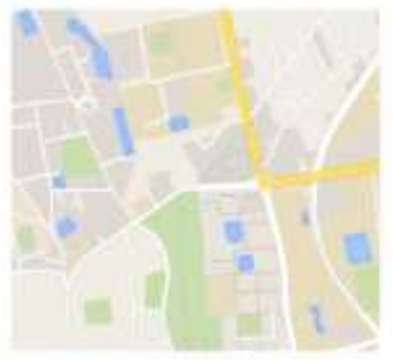

g)

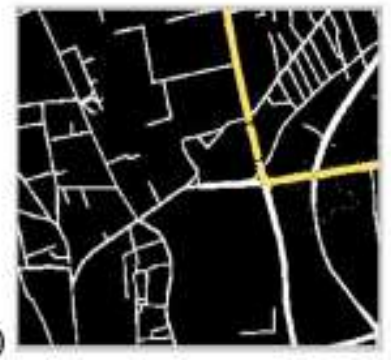

h)

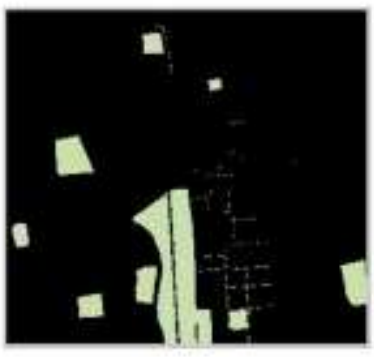

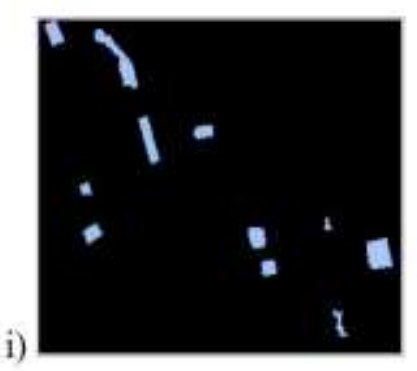

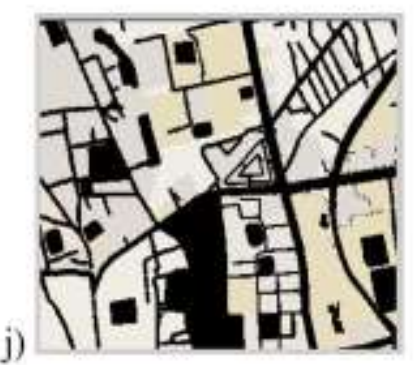

Figure 3. (a, f) Satellite Map, (b, g) Processed Map Showing Only Roads, (c, h) Processed Map Showing Only Fields, (d, i) Processed Map Showing Only Water Bodies, and (e, j) Processed Map Showing Remaining Land Area

One of the strong features of this approach is that it can handle images at any magnification level. Since typical interactive map exhibits a scale bar that changes with magnification level, images can be handled similarly regardless of magnification if the corresponding scale bar is provided for image processing. Once the input image is processed, the program generates separate maps for roads, fields, and water ways as 
shown in Figure 3. Figures 3(a) and 3(f) are the raw map images that can be processed to segment out specific region such as streets as shown in Figures 3(b) and 3(g). Similarly, open fields and water bodies are segmented in Figures 3(c, h) and 3(d, i), respectively. In addition, remaining occupied area such as buildings, parking lots, yards are also segmented as shown in Figures 3(e) and 3(j).

After the maps are being generated the program starts the calculation based on the input scale image and the initial map image. The scale image is now introduced in the calculation to get the accurate dimension of the map used so far. This code compares the pixel size of the scale bar to the value of the scale bar used as input (Figure 2). This provides the real size of the area represented by each pixel in the map. Utilizing some basic MATLAB function such as regionprop, total pixel area of each type of regions (streets, fields etc.,) can be calculated. Utilizing the already calculated area corresponding to each pixel, total area of the regions can be assessed. After calculating the areas of different regions it displays the data as pie chart and bar chart as below (Figure 4).

a)
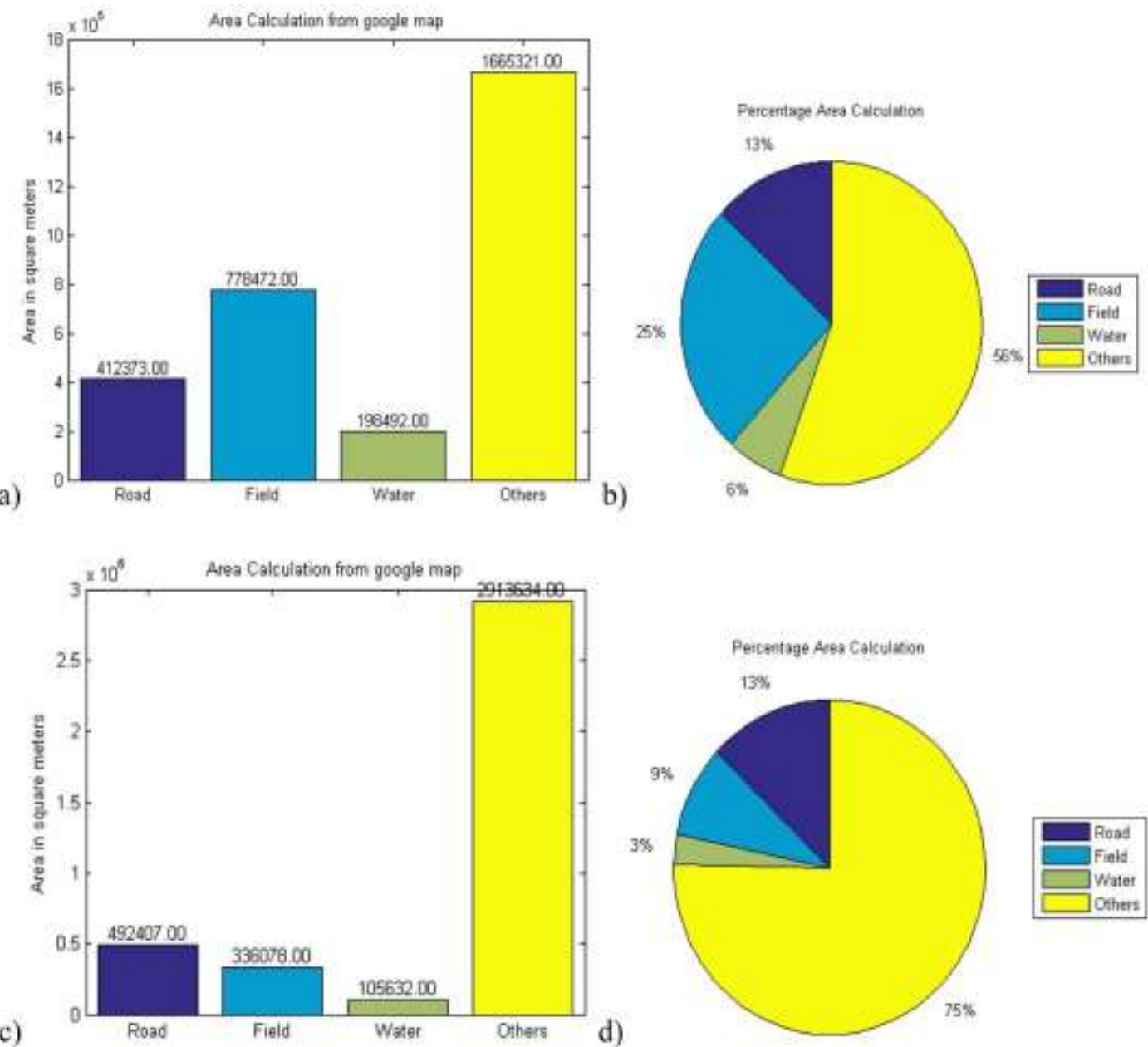

Figure 4. (a) Bar Chart Showing the Total Area of Each Type of Regions for Image 3(a), (b) Pie Chart Shows the Percentage of Total Area Occupied by Each Section for Image 3(a). (c, d) Shows Similar Data for Image 3(f)

For this case, a pie chart exhibits how much area of a certain region is occupied by roads, fields, water bodies, individually (Figure $4 \mathrm{~b} \& \mathrm{~d}$ ). Their approximate areas are also shown in bar charts (Figure 4a \& c). Raw data is also saved in excel file for further use. However, these options can be changed by the users based on their requirements. Presently the program only provides the area statistics on the maps that has been extracted 
but it can be modified to attain data based on many other geometrical properties. The regional data achieved from the maps through this method are quite accurate.

\section{Conclusion}

This study proposes a suitable method of image segmentation to extract geographical map and information out of normal satellite map images. The executable file associated rule out the necessity of technical software like MATLAB and the user friendly graphical interface provides a platform to the users who lack proper computational language expertise. This algorithm generates very accurate results and is pretty flexible for further changes. Some other straightforward segmentation techniques like K-mean segmentation, Canny edge base segmentation, and Watershed segmentation were also applied for the same purpose, however, this technique provided the most satisfactory and accurate segmentation. This program can further be used for urban planning, rural development, annual surveys and any landscape planning and development.

\section{Acknowledgement}

The authors gratefully acknowledge the financial support by BUET Chemical Engineering Forum (BCEF) during this work.

\section{References}

[1] F. Cloppet and A. Boucher, "Segmentation of complex nucleus configurations in biological images", Pattern Recognition Letters, vol. 31, (2010), pp. 755-761.

[2] M. W. Khan, "A survey: Image segmentation techniques", International Journal of Future Computer and Communication, vol. 3, no. 89, (2014).

[3] U. Zakir, A. Leonce and E. Edirisinghe, Proceedings of the 11th Iasted International Conference on Computer Graphics and Imgaing, Innsbruck, Austria, (2010).

[4] K. K. Wong, Z. Sun, J. Tu, S. G. Worthley, J. Mazumdar and D. Abbott, "Medical image diagnostics based on computer-aided flow analysis using magnetic resonance images", Computerized Medical Imaging and Graphics, vol. 36, (2012), pp. 527-541.

[5] F. P. Oliveira and J. M. R. Tavares, "Medical image registration: a review", Computer methods in biomechanics and biomedical engineering, vol. 17, (2014), pp. 73-93.

[6] J. Rittscher, "Characterization of biological processes through automated image analysis", Annual Review of Biomedical Engineering, vol. 12, (2010), pp. 315-344.

[7] M. C. Weiger, S. Ahmed, E. S. Welf and J. M. Haugh, "Directional persistence of cell migration coincides with stability of asymmetric intracellular signaling", Biophysical journal, vol. 98, (2010), pp. 67-75.

[8] N. Sharma and L. M. Aggarwal, "Automated medical image segmentation techniques", Journal of medical physics, vol. 35, no. 3, (2010).

[9] D. B. Bong, K. C. Lai and A. Joseph, "Automatic road network recognition and extraction for urban planning", International Journal of Applied Science, Engineering and Technology, vol. 5, (2009), pp. 209-215.

[10] B. Sowmya and A. Hameed, The National Conference on Communications. (Indian Institute of Technology, Kanpur 2007), pp. 42-47.

[11] B. Sowmya and S. Bhattacharya, in 2005 Annual IEEE India Conference - Indicon, (2005), pp. 41-45.

[12] T. R. Mangala and S. Bhirud, "A new automatic road extraction technique using gradient operation and skeletal ray formation”, International Journal of Computer Applications (0975-8887), (2011).

[13] R. Maurya, P. Gupta and A. S. Shukla, in Image Information Processing (ICIIP), 2011 International Conference on. (IEEE, 2011), pp. 1-6.

[14] M. K. Kerr and G. A. Churchill, "Statistical design and the analysis of gene expression microarray data", Genetical research, vol. 77, (2001), pp. 123-128.

[15] C. J. Veenman, M. J. Reinders and E. Backer, "Resolving motion correspondence for densely moving points", IEEE Transactions on Pattern Analysis and Machine Intelligence, vol. 23, (2001), pp. 54-72.

[16] S. Ahmed, H.-k. Yang, A. E. Ozcam, K. Efimenko, M. C. Weiger, J. Genzer and J. M. Haugh, "Poly (vinylmethylsiloxane) elastomer networks as functional materials for cell adhesion and migration studies", Biomacromolecules, vol. 12, (2011), pp. 1265-1271.

[17] J. Inglot, Advanced image processing with MATLAB, (2012). 
[18] E. S. Welf, S. Ahmed, H. E. Johnson, A. T. Melvin and J. M. Haugh, "Migrating fibroblasts reorient directionality by a metastable, PI3K-dependent mechanism", The Journal of cell biology, vol. 197, (2012), pp. 105-114.

[19] Y.-H. Wang, “Tutorial: Image Segmentation”, National Taiwan University, Taipei, (2010), pp. 1-36.

[20] Y. Han, W.-W. Wang and X.-C. Feng, "A new fast multiphase image segmentation algorithm based on nonconvex regularizer", Pattern Recognition, vol. 45, (2012), pp. 363-372.

[21] R. C. Gonzalez, "Digital Image Processing", (Pearson Education), (2009).

[22] S. T. Smith, "MATLAB: advanced GUI development", (Dog ear publishing), (2006).

[23] C. Depcik and D. N. Assanis, "Graphical user interfaces in an engineering educational environment", Computer Applications in Engineering Education, vol. 13, (2005), pp. 48-59. 